\title{
From Autonomic Nervous System Evaluation to a Novel Paradigm for Scientific Research, Clinical Practice and Economic Development
}

\author{
Ivan Corazza $^{1}$ (D) Walter Struhal ${ }^{2} \cdot$ Christoph $^{\text {Mahringer }}{ }^{2} \cdot$ Giorgio Barletta $^{3,4}$. \\ Pietro Cortelli ${ }^{3,4}$
}

Received: 27 May 2016 / Accepted: 8 August 2016 / Published online: 13 August 2016

(C) Springer Science+Business Media New York 2016

In recent years, the Autonomic Nervous System (ANS) Evaluation Unit of the University of Bologna and the medical physicists of the School of Medicine have worked together on a project to devise a new integrated approach to investigate the cardiovascular reflexes and study the ANS. Our work stemmed from an awareness of the needs of European ANS units [1], the commercial solutions currently available and the different strategies adopted by researchers and clinicians working on this important topic.

Up to now, the clinical approach to ANS evaluation has featured distinct national differences based on the needs and settings of national neurology curricula [2]. As a result, ANS laboratories lacked standardized procedures and were unable to share results with colleagues. Increasing research in the ANS field and the broad spectrum of sensory medical devices available on the market for the evaluation of biosignals have led to a wide variety of instrumentation in individual laboratories. These devices tend not to be interconnected by default

This article is part of the Topical Collection on Systems-Level Quality Improvement

Ivan Corazza

ivan.corazza@unibo.it

1 Experimental, Diagnostic and Specialty Medicine Department, University of Bologna, Bologna, Italy

2 Department of Neurology and Psychiatry, Allgemeines Krankenhaus der Stadt Linz, Linz, Austria

3 Department of Biomedical and Neuromotor Sciences, University of Bologna, Bologna, Italy

4 IRCCS, Institute of Neurological Sciences of Bologna, Bologna, Italy thereby increasing complexity, decreasing usability and leading to isolated applications. All these factors hinder harmonization and standard clinical practice.

The marketing strategies of biomedical manufacturers have been designed to build complete devices able to acquire the signals of interests with powerful algorithms and user-friendly interfaces to facilitate the use of such instrumentation both for clinical routine and research purposes. Although this approach benefits medical staff in ANS units, it has some limitations. First of all, these systems are closed and it is very difficult for the manufacturers to follow rapid research innovations which require ongoing upgrades of hardware and software. Secondly, since these devices are "closed", algorithms and the procedures implemented cannot be custom-made at least for research purposes. Thirdly, the instrumentation is expensive and hence not available to all ANS laboratories worldwide. Lastly, buying these new tools inevitably means disposing of the old devices without being able to use both.

For these reasons, our proposal was both "conservative" and "innovative": our idea was not to get rid of any existing instrumentation but to develop an adaptive comprehensive toolbox of data acquisition and biosignal analysis able to integrate all the existing solutions and improve them by adding new devices. Thanks to the collaboration with a small external manufacturer, the ANScovery system was built and marketed [3].

The strong points of our approach are clear. The proposed solution is open and each laboratory can personalise the experimental setup and the analysis algorithms. Customization can involve different market players so as to stimulate economic and technological partnerships among different manufacturers. This offers an interesting perspective for all small companies which do not have the strength of large multinational corporations and can find in commercial agreements the right way to grow and innovate. 
Our idea allows for further improvements and can lead to a truly new and innovative model. Nowadays, without any direct financial support by a large company the research results of a small laboratory are destined to remain closed and sometimes disappear after one or two papers published in an international journal. In this difficult setting, a novel approach and innovative agreement between researchers and manufacturers is mandatory. Starting from our positive initial experience, our idea can be generalized and extended to other fields of medical practice and research.

Our basic proposal sits astride two different approaches: one based on a fully private medical device and the other totally open. The starting point, based on the Anscovery experience [3], is installing a medical modular system in a laboratory. Sometimes it is very easy to create a modular platform because all the specific instrumentation is already present and only the "container", able to link everything together, is missing. This first step can be taken by clinicians, technicians and private companies already involved in developing instrumentation for the specific purpose of interest. This was the starting point of our past experience but may also be the right approach in different clinical fields.

Then, the modular system can become a powerful platform with all the devices and software routines useful for clinical practice but with the possibility to add external compatible hardware modules. Up to now, the possibility to add new software procedures has been left to the manufacturer, but we believe that the next step to make the system really powerful is to permit the user to add software modules too. Since the common goal should be the improvement of clinical testing and allowing patients to be tested and treated in the best way, all the other users should be able to install the new routines and devices. This will achieve an additional goal: the possibility of different research centres using the same platform to work together and improve their protocols. A possible solution is a software interface for a scripting language and a closed website allowing users to share their scripts. The website can be accessed through periodical subscriptions and if the software is shared on a routine basis, it will be the manufacturer's responsibility to find the best way of compensating the developer.

Nowadays some free and powerful repository of biological signals and software routines are available (i.e. PhysioNet, PhysiotoolKit [4]). Despite the demonstrated usefulness and the powerful of such packages, their main goal is the improvement of scientific research and the result of signal analysis depends on the scientist who developed the scripts, without any external supervisor able to link together the research groups and the results of each of them. Moreover, such solutions do not take into account first fundamental step: the signals acquisition.
This last feature is demanded to the researchers who work separately with all the problems of signals integration previously presented. Our proposal overcome all this difficulties, making the manufacturer of the platform together with the ones of the singles sensors, in a supervising position, and giving the chance, through a dedicated commercial network, to spread the results of each laboratory. Nowadays, the main way to integrate research and daily clinical practice is through a commercial pathway and our approach is coherent with this last issue.

The benefits of such a solution for all those involved are clear:

- An improved modular system will make it more attractive for other laboratories.

- The creation of a wide research network will improve clinical outcomes giving all the groups involved the possibility to have an immediate positive outcome of their daily activities.

- A more prosaic but no less important result will be the dissemination of research results in international scientific journals.

- Since the final solution is the result of integrating different hardware and software devices, economic and technological partnerships can form among the different companies, further improving the system.

The good results of this novel approach depend on the feedback of the manufacturers involved. We think that the interaction between the economic interests of the businesses and the scientific and clinical interests of the users will yield positive results both for them and for the real end-users of our laboratories: the patients. The direct integration between clinical and industrial activities with the creation of wide and powerful networks of researchers can accelerate the benefits for health systems and patients alike.

\section{References}

1. Lahrmann, H., Magnifico, F., Haensch, C.A., and Cortelli, P., Autonomic nervous system laboratories: A European survey. Eur. J. Neurol. 12:375-379, 2005.

2. Struhal, W., Sellner, J., Lisnic, V., Vécsei, L., Müller, E., and Grisold, W., Neurology residency training in Europe-the current situation. Eur. J. Neurol. 18(4):e36-e40, 2011.

3. Corazza, I., Barletta, G., Guaraldi, P., et al., A new integrated instrumental approach to autonomic nervous system assessment. Comput. Methods Prog. Biomed. 117(2):267-276, 2014.

4. Goldberger, A.L., Amaral, L.A.N., Glass, L., et al., PhysioBank, PhysioToolkit, and PhysioNet. Components of a new research resource for complex physiologic signals. Circulation. 101:e215e220, 2000 . 